\title{
Discrimination among schematic stimuli as a function of response mode, constraint redundancy and form of Markov rule
}

$B I L L R$. BROWN and DONALD $F$. DANSEREAU, Texas Christian University, Fort Worth, Tex. 76129

This experiment demonstrated that $S s$ can successfully discriminate among VARGUS 7 stimuli having different central tendencies. Moreover, neither samedifferent classification responses nor similarity judgments varied as a function of the particular Markov prototypes used in the discrimination task. The results thus suggest that the three prototypes were equally encodable, and that the schema chusters were subjectively equidistant, regardless of the amount of noise introduced into the stimuli.

An extensive amount of research has been conducted using the Markov stimuli generated by the VARGUS 7 computer system (Evans, 1967a). The VARGUS 7 program produces histoform stimuli which are deviations of a prototype (schema). The prototype consists of a most probable sequence of column heights, as determined by the transitional probabilities of a seven-element Markov process. A population of instances, all of which are deviations from the same prototype, is termed a schema family (Evans, 1967b). Different prototypes or schemata can be used without affecting the statistical properties underlying the generation procedure.

Although Ss are able to distinguish among VARGUS 7 stimuli sampled from different schema families (Bersted, Brown, \& Evans, in press; Brown \& Evans, 1969), it has not been established that different schema rules are equally difficult with respect to accuracy of Ss' judgments. Specifically, if one conceives of the instances clustered in a multidimensional attribute space, the physical (as well as subjective) distance between instances from any two schema families may be less than that between stimuli belonging to other schema clusters. Furthermore, some prototypes may be characterized by physical properties which make them easier to encode in comparison to other schemata. In either of these cases, variables frequently manipulated in VARGUS 7 discrimination tasks conceivably could be a function of the particular schema populations sampled.

The Markov properties make it extremely difficult to compute a psychologically meaningful measure of the physical distance among VARGUS 7 stimuli. Consequently, the following descriptive measures were considered: (1) the sum of the absolute values of the column height differences computed between adjacent pairs of columns for a prototype, and (2) the length of the largest runs of positive and negative values associated with these differences in column heights for a prototype. These measures are roughly analogous to the angular-variability measure discussed by Brown \& Owen (1967).

The above measures were computed for three nonoverlapping prototypes which have been used extensively in mixed-schemata discrimination tasks. These prototypes can be found in Bersted, Brown, \& Evans (1968), and are identified as Schemata 2,3 , and 4 . Schemata 2 and 3 were almost identical in terms of the two measures; Schema 4, on the other hand, was dissimilar to the other two prototypes with respect to both measures. The means and variances of the column heights were equal for all prototypes.

The present experiment was conducted to determine the extent to which classification responses and similarity judgments of paired VARGUS 7 stimuli, generated at three different levels of constraint redundancy (Rc), vary as a function of the particular schema families sampled. On the basis of the two measures discussed above, it was hypothesized that (1) paired instances sampled from Schema Families 2 and 3 would be judged more similar, and would receive more incorrect same classifications, than trials involving pairings of stimuli from Schema 4 with either Schemata 2 or 3 ; and (2) discrimination performance should be better on pairings of instances sampled from Schema Family 4 (in comparison to stimulus pairings involving either of the other two schemata). The latter prediction was based on the observation that the magnitude of differences between adjacent column heights for Schema 4 was greater than for the other Prototypes. Using a task requiring predictions of sequentially presented Markov stimuli, Dansereau \& Brown (1969) demonstrated that large deviations in pairs of column heights were more easily learned than small deviations.

$$
\text { SUBJECTS }
$$

The Ss were 48 undergraduate students enrolled in introductory psychology courses at Texas Christian University.

\section{STIMULI}

The stimuli were 24 column histoforms generated by the VARGUS 7 computer program at $50 \%, 70 \%$, and $100 \%$ Rc. The Rc variable refers to the extent to which the stimuli adhere to a schema rule (Evans, 1967c). Three distinct families of instances were produced by creating distortions of the three most probable sequences of column heights (i.e., Schemata 2,3, and 4) discussed previously.

PROCEDURE
The Ss were given 60 trials by means of a Kodak Carousel slide projector. On a typical trial, 24 of the Ss (8 Ss per Rc condition) viewed two stimuli and then judged them as belonging to the same or different schema families; the remaining 24 Ss ( 8 Ss per Rc condition) judged the same pairs of patterns as to their degree of similarity on a 20-point scale. Fifty per cent of the 60 trials involved the pairing of instances from different schema families (different trials), whereas the remaining trials involved the pairing of instances from the same schema family (same trials). The Ss were permitted to view each pair of instances for $8 \mathrm{sec}$; the stimuli were then removed from the screen, and Ss were given $7 \mathrm{sec}$ in which to mark their responses.

\section{RESULTS}

A two-way analysis of variance, with one between-group factor (magnitude of $\mathrm{Rc}$ ) and one within-group factor (same vs different trials), was performed, with total correct classification responses as the dependent variable. There was a significant $R c$ main effect $[F(2,21)=12.99, p<.01]$ and a significant same-different trials main effect $[F(1,21)=4.32, p<.05]$. Increasing the magnitude of $R c$ facilitated performance on both same and different trials. Performance was higher on different trials than it was on same trials for all $\mathrm{Rc}$ conditions.

An analysis identical to that discussed above was performed, using total similarity judgments as the dependent variable. There was a highly significant same-different trials effect $[F(1,21)=77.85, \quad p<.001], \quad a$ significant Rc by Same-Different Trials interaction $[F(2,21)=19.27, p<.001]$, and a significant simple main effect of $\mathrm{Rc}$ for same trials $[F(2,21)=22.97, p<.001]$. The simple main effect of $\mathrm{Rc}$ for different trials was not significant. Figure 1 shows that mean similarity judgments for all Rc groups were higher on same trials than on different trials. Increasing the magnitude of Rc produced higher similarity ratings with stimuli sampled from the same schemata, but did not differentially affect similarity judgments of instances from different schema families.

In order to determine if instances sampled from the three schema families differed with 


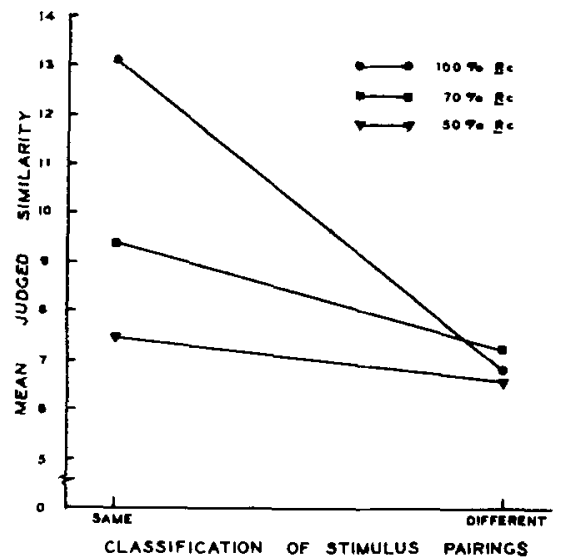

Fig. 1. Mean judged similarity as a function of classification of stimulus pairings and $R c$ conditions.

respect to how accurately they were classified, two-way analyses of variance, with one between-group factor (Rc magnitude) and one within-group factor (schema pairings), were performed separately for same and different trials. The dependent variable in both analyses was the total number of correct classification responses. The analysis for both same and different trials showed that neither the schema pairing factor nor the Rc by schema pairings was significant. Only $18 \%$ of the total within-group variance on same trials was accounted for by these two effects combined, while the effects accounted for $23 \%$ of the within-group variance on different trials.

Analyses identical to the ones discussed in the previous paragraph were performed with total similarity ratings as the dependent variable. As with classification responses, neither the schema-pairings main effect nor the $\mathrm{Rc}$ by Schema Pairings interaction was significant (only $2 \%$ of the total within-group variance was accounted for by these effects on same trials, and $15 \%$ of the total variance was accounted for on different trials). Consequently, regardless of the magnitude of Rc, accuracy of classification responses and judged similarity did not significantly vary as a function of the particular schema families sampled.

\section{DISCUSSION}

The results reported here support the notion that, regardless of the response mode used, Ss can successfully discriminate between instances having different central tendencies. Increasing the magnitude of Rc facilitated classification performance on both same and different trials. Similarly, increasing $\mathbf{R c}$ produced an increment in mean judged similarity of paired stimuli sampled from the same schema family, although the Rc variable had no effect on similarity ratings of stimuli on different trials. The latter result may be attributed to the fact that, as the magnitude of stimulus distortion increased, Ss tended to use a smaller proportion of the 20-point rating scale. The absolute scale, in fact, shifted towards the "very dissimilar" point of the scale as Rc was decreased.

The fact that classification performance was higher on different than on same trials may be attributed primarily to Ss making more different than same responses. One possible explanation for the existence of such a bias is that $S$ s were explicitly forming more stimulus categories than were schema families (i.e., several categories may reflect each schema separately); this suggestion receives support from a study, conducted by Bersted, Brown, \& Evans (in press), which showed that Ss actually form subcategories when they are not told the number of schema families represented in a sorting task.

Contrary to the hypotheses, classification performance and similarity judgments did not significantly vary as a function of the particular schemata utilized in the present study. The fact that performance did not vary as a function of the different schema pairings thus suggests that (1) Ss made their judgments within a measurement space that reflected equal distances among the schema clusters (i.e., they attended to pattern features different from the configurations used in computing the proposed measures); or (2) Ss were responding within the measurement space represented by the computed measures, but the differences among the three prototypes were not sufficiently large to create disparity in classification responses and similarity judgments.

The fact that performance on same trials was not differentially influenced by the schema rules supports the notion that the three prototypes were equally encodable. These results, coupled with the suggestion that the schema clusters are subjectively equidistant, are clearly relevant to the interpretation of the effects of several empirical conditions manipulated in other VARGUS 7 experiments. For example, Brown \& Evans (1969) demonstrated performance in a two-schemata task (using Schemata 2 and 3) to be superior to that exhibited in a task requiring $S$ s to discriminate among instances sampled from three schema families (Schemata 2, 3, and 4). One explanation for such an effect which has been considered is that the prototypes for Schema Families 2 and 3 were subjectively very similar to each other, but both were highly dissimilar to Schema 4 (a suggestion supported by the two prototype measures discussed previously). Given a set number of trials, performance would thus be higher in the three-schemata task than in the two-schemata experiment. The results of the present study clearly fail to support the above hypothesis with respect to the three levels of Rc and both response modes.

Since the results concerning the effect of the schema-pairings variable, in fact, constituted an acceptance of the null hypothesis, one should be extremely cautious in making general conclusions about these findings. The generality of the results is further limited by the fact that only three schema populations were sampled. Instances from these three schema families, however, have been used in a large number of pattern-discrimination experiments, and are representative of other stimulus populations that can be produced by the present VARGUS 7 system.

\section{REFERENCES}

BERSTED, C. T., BROWN, B. R., \& EVANS, S. H. A standard set of VARGUS 7 patterns at three levels of schematic redundancy. Psychonomic Monograph Supplements, 1968, 2(13, Whole No. 29), $251-282$.

BERSTED, C. T., BROWN, B. R., \& EVANS, S. H. Free sorting of stimuli clustered in a multidimensional attribute space. Perception \& Psychophysics, in press.

BROWN, B. R., \& EVANS, S. H. Perceptual learning in pattern discrimination tasks with two and three schema categories. Psychonomic Science, 1969, 15, 101-103.

BROWN, D. R., \& OWEN, D. H. The metrics of visual form: Methodological dyspepsia. Psychological Bulletin, 1967, 68, 243-259.

DANSEREAU, D. F., \& BROWN, B. R. Human and machine recognition of sequentially presented Markov patterns. Paper presented at annual convention of the Southwestem Psychological Association, April, 1969.

EVANS, S. H. VARGUS 7: Computed patterns from Markov processes. Behavioral Science, 1967a, 12, 323-328.

EVANS, S. H. A brief statement of schema theory. Psychonomic Science, 1967b, 8, 87-88.

EVANS, S. H. Redundancy as a variable in pattern perception. Psychological Bulletin, 1967c, 67, 104113.

NOTE

1. This research was conducted by the Institu te for the Study of Cognitive Systems and supported by a Department of Defense Project THEMIS contract, No. DAD005-C00176, under the Department of the Army. 